\title{
Observations on entomopathogenic fungus Ophiocordyceps neovolkiana on Coconut root grub Leucopholis coneophora
}

\author{
P. K. Laya ${ }^{1}$, C. K. Yamini Varma ${ }^{2}$ K. Anita Cherian ${ }^{3}$, M. M. Anees ${ }^{4}$ and C. R. Rashmi ${ }^{5}$ \\ ${ }^{1,3,4}$ Department of Plant Pathology, College of Horticulture, Vellanikkara 680656, Kerala, India \\ ${ }^{2}$ Department of Plant Pathology, AICRP on Spices, Pepper Research Station, Panniyur 670142, Kerala, India \\ ${ }^{5}$ Department of Vegetable Science, AICVIP, College of Horticulture, Vellanikkara 680656, Kerala, India \\ *Corresponding authorEmail: mail2layapk@gmail.com
}

Submitted on August 20, 2019; Accepted on November 19, 2019)

\section{ABSTRACT}

\begin{abstract}
Ophiocordyceps are entomopathogenic fungi on arthropods which parasitize, kill and mummify hosts and produce fruit bodies out of them. Due to the presence of many bioactive compounds like adenosine, cordycepin, ergosterol, these fungi have been used as food as well as medicine against many diseases. Even though cosmopolitan in distribution, many Ophiocordyceps like $O$. sinensis are mostly confined to high Himalayan Mountains in India, China, Tibet, Nepal, Bhutan at an altitude of 3000 to 5000 msl. Owing to high price, Cordyceps are known as "Himalayan gold". From surveys conducted in coastal sandy areas of Kasargod District (Kerala), it was observed that Ophiocordyceps sp. attacks coconut root grubs (Leucopholis coneophora Burm.), which is an endemic pest in the sandy tracts. The fungus was isolated in potato dextrose agar medium. Detailed morphological studies have been carried out. The molecular characterization showed homology with Ophiocordyceps neovolkiana (Kobayasi) strain KC1 with $98 \%$ identity. The ITS sequence has been deposited at NCBI with accession \# MH668282 and culture has been deposited in the National Fungal Culture Collection of India with herbarium \# NFCCI 4331.
\end{abstract}

KEYWORDS: Cordyceps, isolation, morphology, coconut root grub, coastal sandy region, southwest India, Kasargod

\section{INTRODUCTION}

Ophiocordyceps is one of the most valued and best known fungal genus in traditional Chinese Medicine. Even though the stromatal heads of Ophiocordyceps resembles mushrooms, it is an ascomycetous entomopathogenic fungus (Kingdom Fungi, Phylum Ascomycota, Subphylum Pezizomycotina, Class Sordariomycetes, Subclass Hypocreomycetidae, Order Hypocreales, and Family Ophiocordycipitaceae). Based on molecular phylogenetic study, Sung et al. (2007) separated the mega genus Cordyceps into four genera, viz. Cordyceps, Ophiocordyceps, Metacordyceps and Elaphocordyceps. As a result, members like $C$. sinensis and related organisms were transferred to the genus Ophiocordyceps, hence renamed as $O$. sinensis. The name Cordyceps comes from two Latin words: cord and ceps, meaning "club" and "head", respectively (Holliday and Cleaver, 2004). It parasitizes the body of the insect host and utilizes the internal tissues as a substrate for its growth, followed by complete replacement of internal tissues by mycelium, which finally get converted into a sclerotium. Later from the sclerotial bodies, stroma of the fungus emerges above the ground, thus it is commonly called as "Caterpillar fungus". It is collected along with the sclerotium for medicinal and other uses (Li et al., 2011). Due to strict parasitism and specific geographic environment in which it grows, the output of natural Ophiocordyceps cannot meet market demand and thus its price is unusually high. In the Indian markets this fungus is valued as much as Rs. 15 lakhs $/ \mathrm{kg}$ in recent years. Most of the bioactive compounds of Ophiocordyceps sp. have been exploited for use in traditional and modern ethno-medicine, for treatment of various diseases like cancer, diarrhoea, headache, muscle pain and so on.

From the surveys conducted in the coastal sandy areas of Kasargod district, it was observed that entomopathogenic species of Ophiocordyceps was emerging during southwest monsoon (June-August) from the infected coconut white root grub (Leocopholis coneophora Burm.), which is an endemic pest in the coastal sandy tracts of Kasragod District (Kumar and Aparna, 2014). So far there are no systematic studies conducted on the mycological aspects of Ophiocordyceps sp. from Kerala and hence a detailed investigation was carried out focusing on the isolation, identification and characterization of Ophiocordyceps sp.

\section{MATERIALSAND METHODS}

Survey: Surveys were carried out for the occurrence of fruit bodies of Ophiocordyceps in Padannakkad, Valiyaparamba and Pilicode area of Kasargod district of Kerala, during June to August months of 2017 and 2018. Observations of the fruit bodies were made. The fruit bodies were collected in brown paper covers and brought to the laboratory for further observations. These fruit bodies were preserved fresh as well as in dry forms.

Isolation of the fungus: Isolation of fungus was carried out from different parts of the fungal structures like sclerotia, stipe and stroma. The isolation was performed by following the standard tissue culture technique. The samples were washed in running tap water. From all the three parts (sclerotia, stipe and stroma), tissues were taken for isolation. These tissues were cut into small pieces using a sterile blade, and subsequently cut small pieces were disinfected with mercuric chloride $(0.1 \%)$ for one minute. After three washings using sterilized distilled water, the samples were placed on solidified potato dextrose agar (PDA) medium aseptically. After incubation at room temperature $\left(26 \pm 2^{\circ} \mathrm{C}\right)$, the fungal growth from second to sixth days of incubation was subsequently sub-cultured to solidified PDA in sterile Petri dishes. Purification of isolate was achieved by periodic subculturing and maintenance of the isolate was done in PDA slants under refrigerated condition at $4^{\circ} \mathrm{C}$ for further studies.

Morphological studies and identification: Morphological studies (macroscopic and microscopic) of the fungus were 
carried out. Various characters like shape, size and colour of sclerotia, stipe and stroma and branching pattern of the stroma associated with the insect-fungal complex were studied and observations were recorded.

Microscopic slides were prepared using lactophenol cotton blue stain and observed under compound microscope at $5 \mathrm{x}$, 10x, 40x and 100x magnifications to study the anamorphic stage including hyphae, synnemata, conidiophores and conidia and teleomorphic stage including perithecia, asci and ascospores of the fungus. Measurements and photomicrographs were taken using Zeiss Axiolab image analyzer.

Molecular characterization of the isolate was done to identify the isolate at species level by DNA barcoding using universal primers of ITS sequences. Sequence analysis and nucleotide homology of the fungus was analysed through the BLAST programme of NCBI (http:// ncbi.nlm.nhm.gov/blast). Sequence analysis of the culture showed homology with Ophiocordyceps neovolkiana having 98 per cent identity. The sequence of the fungus was deposited at the GenBank of NCBI with accession number MH 668282 and the culture was deposited in National Fungal Culture Collection of India at MACS Agharkar Institute, Pune with culture number NFCCI 4331.

\section{RESULTS}

Survey: Purposive sampling surveys were conducted in three locations of coastal sandy tracts of Kasargod district during June to September months of 2017 and 2018. The fruiting bodies of Cordyceps sp. emerged from the parasitized grub were collected from three locations, viz. Instructional Farm of College of Agriculture, Padannakkad, Valiyaparamba area of Nileshwar and Regional Agricultural Research Station, Pilicode, where the coconut root grub is an endemic polyphagous pest.

The fruit bodies were found to be emerged from the soil after summer showers usually during mid May and after the onset of monsoon showers during early June. The population of fruit bodies in one square meter area of soil were assessed in all the three locations and represented in the Table 1. Among the three areas surveyed, maximum number of fruiting bodies (average of $13.86 \mathrm{~m}^{2}$ ) were found at Instructional Farm of College of Agriculture, Padannakkad, Kasargod.

Table 1: Extent of fruit bodies in three locations (mean of four replications)

\begin{tabular}{|c|c|c|c|}
\hline \multirow{2}{*}{} & \multicolumn{2}{|c|}{ Number of fruiting bodies $\mathbf{~ m}^{\mathbf{2}}$} & \multirow{2}{*}{ Mean } \\
\cline { 2 - 3 } & $\mathbf{2 0 1 7}$ & $\mathbf{2 0 1 8}$ & \\
\hline Padannakkad & 15.25 & 12.50 & 13.86 \\
\hline Valiaparamba & 5.75 & 4.50 & 5.13 \\
\hline Pilicode & 2.75 & 2.25 & 2.50 \\
\hline
\end{tabular}

Isolation: The fungus was isolated on PDAmedium and named as CD1 (Fig. 1a) and was brought into pure culture. The mycelia appeared initially white, later turning to creamish white to salmon colour (Fig. 1b) and the underside of the plate being light brown (Fig. 1c). Subsequently, numerous thread like pinkish synnemata appeared in the culture representing the anamorphic stage Hirsutella sp. The anamorph produced similar type of conidia as produced in the field.

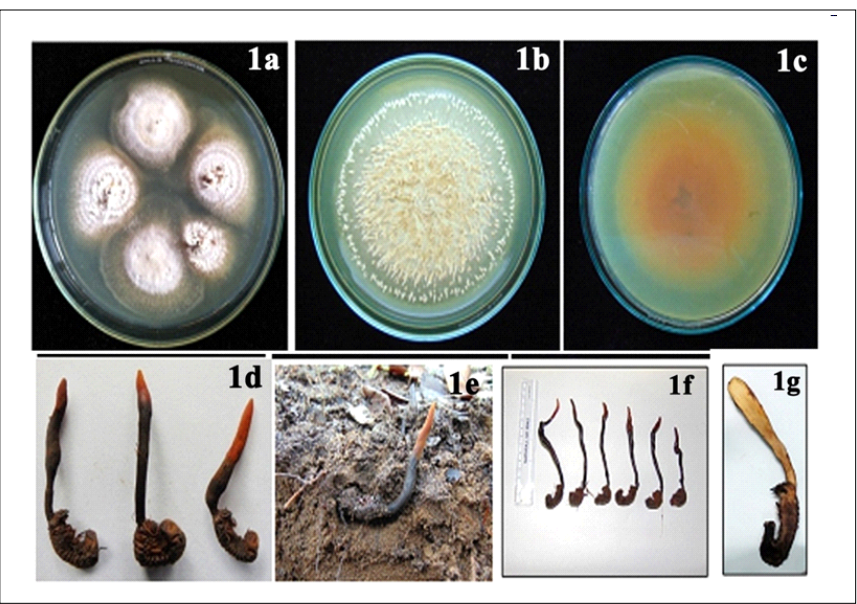

Fig.1a: Typical colonies of Ophiocordyceps sp. on isolation plate;

Fig.1b \& c: Culture plate of Ophiocordyceps sp.;

Fig.1d: AssociationofOphiocordyceps sp. with Leucopholis coneophora;

Fig. 1e: Ophiocordyceps sp. in natural habitat;

Fig. 1f: Variation in size of fruit body;

Fig. 1g: L. S. of Ophiocordyceps sp.

Morphological studies: The fruit body of the fungus was found to be emerging from the cephalic region of the body of third instar grub of Leucopholis coneophora. The clubshaped upper portion of fruit body called as stroma, which is the fertile portion, in which the perithecia were present, the middle stalk-like part, stipe and the mummified dead grub known as sclerotium were observed (Fig. 1d). The stipe and stroma portion of the fruiting bodies were found to be above the soil and infected grub was located at a depth of around 5 to $10 \mathrm{~cm}$ below the soil (Fig. 1e). Average length and girth of the fruiting bodies obtained from all the three locations were measured (Table 2), in which the maximum length of $11.9 \mathrm{~cm}$ and $1.6 \mathrm{~cm}$ for stipe and stroma, respectively were obtained from Instructional farm of College of Agriculture, Padannakkad. Maximum girth of $3.9 \mathrm{~cm}$ and $1.7 \mathrm{~cm}$ for stipe and stroma was also observed from the same soil (Fig. 1f). The longitudinal section of the fruiting body showed complete mycelial colonization of the insect body by the fungus, where the internal organs of the grub were replaced with fungal mycelium (Fig. 1g).

Comprehensive study on the process of fruit body emergence was carried out in all the three locations. From the buried infected grubs, yellowish-orange coloured stalks of about $0.3-0.6 \mathrm{~cm}$ thickness emerged during the last week of May or first week of June and the tip of which later became bulged and turned to bright orange red in colour. Stromatal heads were observed to emerge from the cephalic region of the infected third instar grubs of $L$. coneophora. After 40 days, by the second week of July the stroma region showed numerous humps which were initially orange red in colour turning to brown representing formation of perithecia. The colour turned to dark-brown with the complete maturation of perithecia and the release of ascospores occurred in a period of two weeks. During August, after an interval of two weeks the stroma region became dark-brown to black and numerous white colored hook like sporulating structures called synnemata were developed representing the conidial stage of 


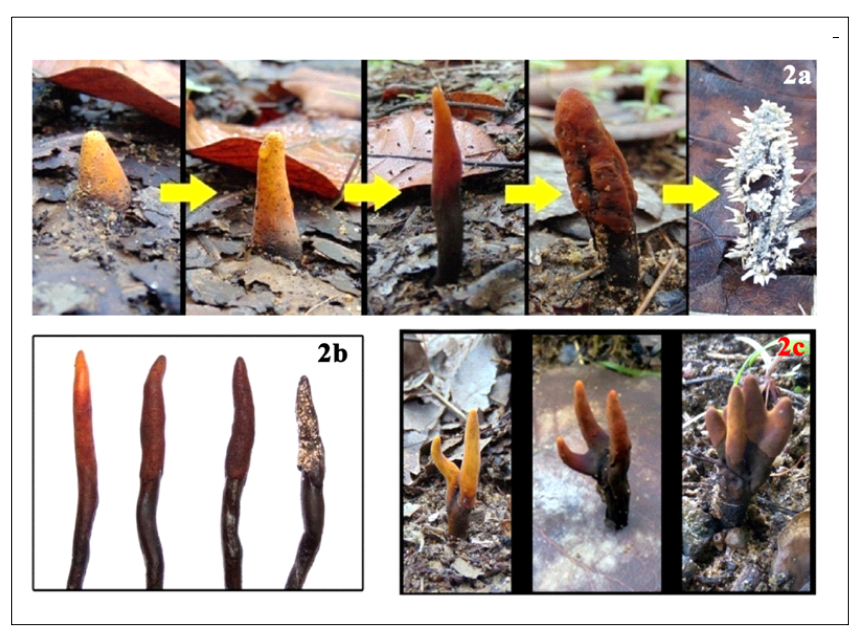

Fig. 2a: Different stages of fruiting body development in the field;

Fig. 2b: Changes in stroma during development;

Fig. 2c: Branching pattern of stroma

the fungus (Hirsutella sp.) (Fig. 2a). Hence, a gradual change of colour and texture of stroma was seen during different stages of development (Fig. 2b). Branching of stroma was noticed as double and very rarely three to five branches from a single stroma were observed (Fig. 2c). After one month, by the second week of September, the whole fruiting body got decomposed by the action of soil dwelling saprophytes. Various other macroscopic characters like shape of the fruit body, colour of sclerotium, stipe and stroma collected from the three locations surveyed were also studied and the observations are shown in Table 2.

Table 2: Macroscopic characters of Cordyceps sp. in three locations (mean of 15 replicates)

\begin{tabular}{|c|c|c|c|c|c|c|c|c|}
\hline & \multicolumn{8}{|c|}{ Characters of fruiting body } \\
\hline & \multirow[t]{3}{*}{ Shape } & \multicolumn{4}{|c|}{ Size (cm) } & \multicolumn{3}{|c|}{ Colour } \\
\hline & & \multicolumn{2}{|c|}{ Length } & \multicolumn{2}{|c|}{ Girth } & \multirow[t]{2}{*}{ Sclerotia } & \multirow[t]{2}{*}{ Stipe } & \multirow[t]{2}{*}{ Sroma } \\
\hline & & Stipe & Stroma & Stipe & Stroma & & & \\
\hline Padannakkad & Spindle & 11.9 & 3.9 & 1.6 & 1.7 & Black & $\begin{array}{l}\text { Dark- } \\
\text { brown }\end{array}$ & $\begin{array}{l}\text { Bright orange turning } \\
\text { to dark-brown }\end{array}$ \\
\hline Valiaparamba & Club & 8.5 & 2.8 & 1.3 & 1.4 & $\begin{array}{l}\text { Dark- } \\
\text { brown }\end{array}$ & $\begin{array}{l}\text { Dark- } \\
\text { brown }\end{array}$ & $\begin{array}{l}\text { Light-brown turning } \\
\text { to dark-brown. }\end{array}$ \\
\hline Pilicode & Club & 10.4 & 3.2 & 1.2 & 1.4 & $\begin{array}{l}\text { Dark- } \\
\text { brown }\end{array}$ & Brown & $\begin{array}{l}\text { Reddish-brown } \\
\text { turning to dark-brown }\end{array}$ \\
\hline
\end{tabular}

Microscopic studies: Microscopic observations of Ophiocordyceps sp. such as characters of anamorphic stage including hyphae, synnemata, conidiophores and conidia and teleomorphic stage including perithecia, asci and ascospores were taken.

Hyphae were hyaline septate and branched with a width of 1.1 to $1.52 \mu \mathrm{m}$ (Fig. 3a). The length and width of synnemata from which the conidiophores were arising was found to be 2136 to $2351 \mu \mathrm{m}$ and 991 to $1100 \mu \mathrm{m}$, respectively (Fig. 3b). Synnemata was found to have cylindrical shape with numerous conidiophores all over the surface. In natural habitat the synnemata started to form initially as white cottony cushion later turning to light pinkish white hook like structures on the stroma having a length of $0.2-1.3 \mathrm{~cm}$ long (Fig. 3c).

The conidia were formed either singly or in chains on long conidiophores measuring 5.64 to $8.78 \mu \mathrm{m}$. The conidiophores can be single or branched. The conidia were dimorphic and hyaline among which one type of the conidia was spherical with a diameter of 1.9 to $5.7 \mu \mathrm{m}$ (Fig. 3e) and the other was spindle shaped with a length of 2.5 to $6.3 \mu \mathrm{m}$ and width of 0.53 to $2.41 \mu \mathrm{m}$ (Fig. 3g). The spherical conidia were arranged singly (Fig. 3d) and spindle shaped conidia were arranged in chains on the conidiophores (Fig. 3f;Table 3).

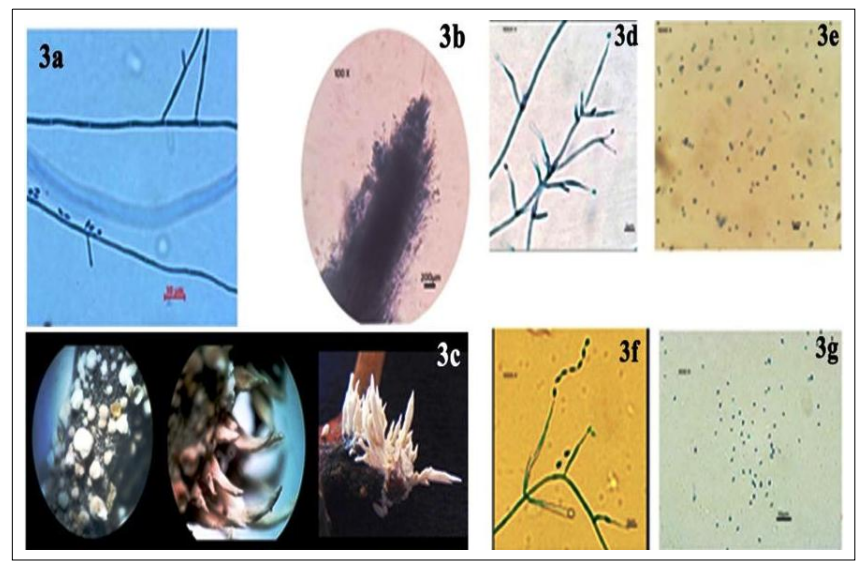

Fig. 3a: Hypha of Hirsutella sp.;

Fig. 3b: Synnemata;

Fig.3c: Different stages of development of synnemata on the fruiting body in natural habitat;

Fig. 3d: Single spherical conidia on conidiophores;

Fig. 3e: Spherical conidia;

Fig. 3f: Spindle conidia in chain on conidiophores;

Fig. 3g: Spindle conidia

Table 3: Microscopic characters of anamorphic stage.

\begin{tabular}{|c|c|c|}
\hline & Description & Dimension $(\boldsymbol{\mu m})$ \\
\hline Hypha & Hyaline, septate and branched & $1.1-1.52$ \\
\hline Synnemata & Cylidrical with numerous conidiophores & $2136-2351 \times 991-1100$ \\
\hline Conidiophore & Elongated, single or branched & $5.64-8.78$ \\
\hline Spherical conidia & Born singly on conidiophore & $1.9-5.7$ \\
\hline Spindle conidia & Born in chains on conidiophore & $2.5-6.3 \times 0.53-2.41$ \\
\hline
\end{tabular}

Microscopic examination of thin cross sections of stroma showed numerous perithecia towards the peripheral regions (Fig. 4a). They were peripheral to slightly immersed globular to oval in shape, narrow towards the tip (Fig. 4b). Openings of perithecia, the ostioles through which release of ascospores to the outside occurs were found at the tip (Fig. 4c). The length and width of perithecia was found to be 82.6 to $396.1 \mu \mathrm{m}$ and 93.6 to $171.3 \mu \mathrm{m}$, respectively. The thickness of the perithecial wall ranged from 16.6 to 21.7 $\mu \mathrm{m}$ (Figs. 4d,e,f,g). On the examination of perithecia numerous elongated asci were observed inside the perithecium. Crozier formation stage during the ascus development was observed in immature perithecium (Fig. 5a). On maturation, asci were observed as a bundle inside the perithecium (Fig. 5b). The ascus was elongated with length ranging from 115.4 to $170.1 \mu \mathrm{m}$ and width of 5.2 to $10 \mu \mathrm{m}$ (Fig. 5c). The tip of the ascus had a prominent cap or operculum, the lid-like structure, through which the ascospores are released out of ascus (Fig. 5d). The ascus possesses eight filiform ascospores (Fig. 5e) which were four-partite with 105.3 to $135.7 \mu \mathrm{m}$ length and $2.5 \mu \mathrm{m}$ to 2.7 $\mu \mathrm{m}$ width (Fig. 5f). The ascospores usually split into four 


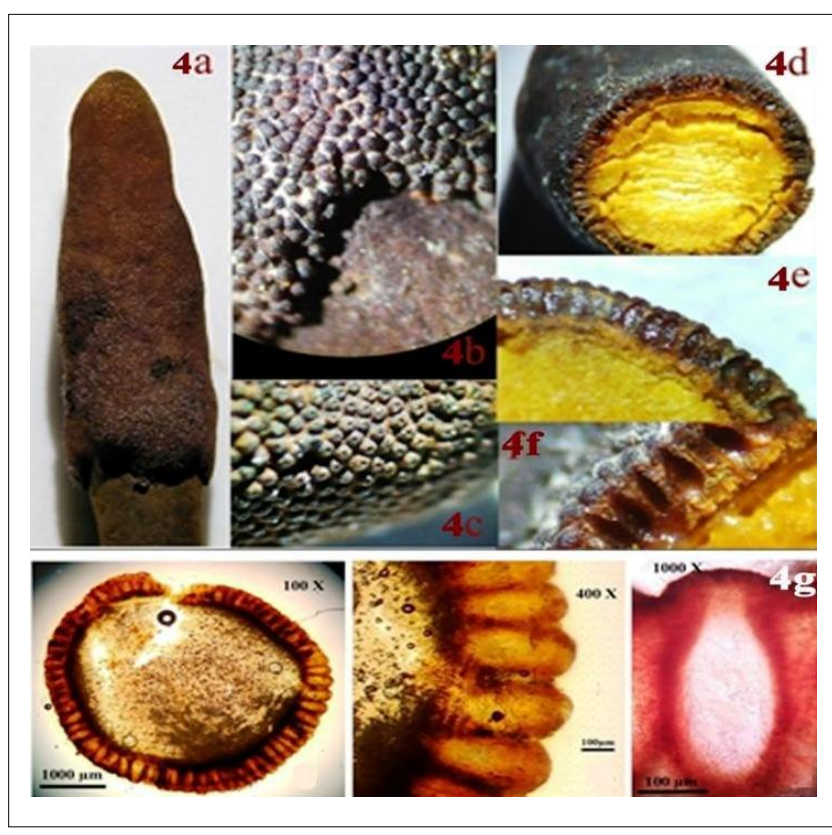

Fig.4a: Stroma;

Fig. 4b: Perithecia;

Fig. 4c: Ostiole;

Fig. 4d: C. S. of stroma;

Fig. 4e: Peripheral arrangement of perithecia;

Fig. 4f: Globular to oval perithecia;

Fig. 4g: Microscopic characters of stroma.

part spores in which the middle two spores were cylindrical and the terminal spores were pointed at the tip, having 25.58 to $44.59 \mu \mathrm{m}$ length and $2.52-2.74 \mu \mathrm{m}$ width (Fig. 5g; Table 4).

\section{DISCUSSION}

Ophiocordyceps sinensis, a well-known and valued traditional Chinese medicinal mushroom, also called Dong Chong Xia Cao (winter worm summer grass), is a parasitic complex of fungus and caterpillar of Hepialis armoricanus, family Hepialidae. Due to the presence of many bioactive compounds like adenosine, cordycepin, ergosterol, Ophiocordyceps have been used as food as well as in herbal medicine to treat respiratory, pulmonary, renal, liver, and cardiovascular diseases, hyposexuality, and hyperlipidemia (Arora, 2015). Even though the distribution of this fungus is cosmopolitan, many of its members like $O$. sinensis are mostly confined to high Himalayan Mountains in China, Tibet, Nepal, Bhutan and India at an altitude of 3000 to $5000 \mathrm{msl}$ (Sharma, 2004). But from the surveys conducted in costal sandy tracts of Kasargod District of Kerala, it was found that Ophiocordyceps sp. parasitizes the third instar grubs of Leucopholis coneophora. The present observations are in accordance with the report of (Kumar and Aparna, 2014). Sreedhar and Karun (2017) reported the presence of Ophiocordyceps nutans associated with sap-sucking pentatomid stinkbug Halyomorpha halys in Western Ghats. Dattaraj et al. (2018) documented reports by various scientists about the occurrence of Cordyceps and allied species in the Western Ghats and southwest coast of India and also reported six species of Cordyceps recovered in the scrub jungles of southwest Karnataka.

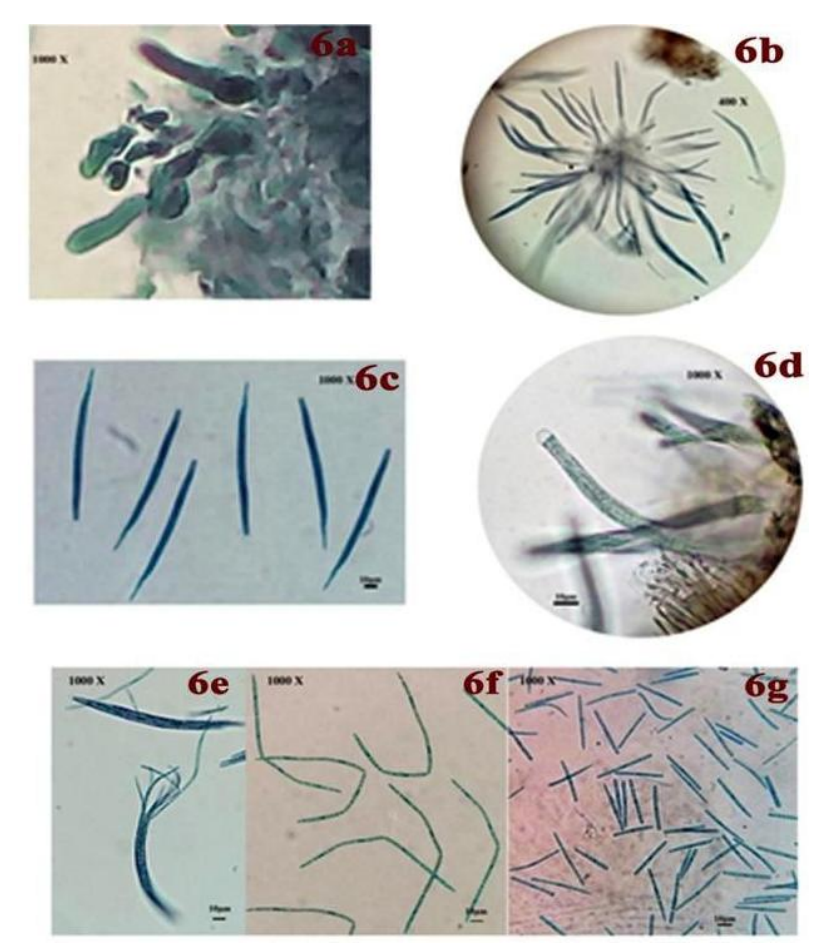

Fig. 5a: Crozier formation during ascus development;

Fig. 5b: Mass of mature asci;

Fig.5c: Asci;

Fig. 5d: Ascus with operculum;

Fig. 5e: Eight ascospores in an ascus;

Fig. 5f: Ascospores;

Fig. 5g: Part spores of ascospore.

Table 4: Microscopic characters of teleomorphic stage

\begin{tabular}{|c|c|c|}
\hline & Description & Diamension $(\boldsymbol{\mu m})^{*}$ \\
\hline Perithecia & Globular -oval with ostiole & $82.6-396.1 \times 93.6-171.27$ \\
\hline Perithecial Wall & Thick & $16.6-21.65$ \\
\hline Ascus & Elongated & $115.4-170.11 \times 5.2-10$ \\
\hline Acsospore & Filiform and four partite & $105.3-135.7 \times 2.51-2.73$ \\
\hline $\begin{array}{c}\text { Ascospore part } \\
\text { spore }\end{array}$ & $\begin{array}{c}\text { Terminal spore pointed at the tip and middle } \\
\text { spores are cylindrical }\end{array}$ & $25.58-44.59 \times 2.52-2.74$ \\
\hline
\end{tabular}

So far there are no systematic studies conducted on the mycological aspects of Ophiocordyceps sp. from Kerala and therefore this study is a first attempt for the isolation and morphological characterization of this fungus. The period of occurrence of Ophiocordyceps sp. as per the sampling surveys conducted in three selected locations of Kasargod district during 2017 and 2018 has similarity with reports of other scientists in different countries. It was reported that the best time of collection of Cordyceps sphecocephala specimens is from July to September in Japan (Kobayasi and Shimizu, 1980) and Korea (Namet al., 2006). The presence of the fungus in tropical conditions at low altitudes of Kerala may be attributed to the high humidity and low temperature situations prevailing in the state during south-west monsoon periods, the time at which the fungus was noticed in the field (Kumar and Aparna, 2014).

In the fruiting bodies of Ophiocordyceps sp. the bulged tip portion was observed as stroma and stalk like structure as stipe and sclerotium was the mummified grub which was 
completely filled with mycelia of the fungus. The tips of perithecia were found as numerous humps which are initially orange red in colour turning to dark-brown. These findings were having similarity with observations of other researchers. The morphological characters of $O$. sinensis was described by Shrestha et al. (2010) as it consists of two parts, a fungal endosclerotium (caterpillar) and stroma which was initially yellow in colour and later turning to dark brown or black, which was longer than the caterpillar itself, usually $4-10 \mathrm{~cm}$. Sung et al. (2010) also reported the morphological characters of the fruiting body of $O$. sinensis. They noted that fruiting body grew singly from the larval head, and was clavate, sublanceolate or fusiform and distinct from the stipe, which was slender, glabrous, and longitudinally furrowed or ridged.

The hyphae of Cordyceps were hyaline, septate and branched with a width of 1.1-1.52 $\mu \mathrm{m}$. Chen et al. (2006) observed similar characters for the hyphae of $C$. sinensis. They noted hyphae as ramose, septate and hyaline. Arora et al. (2013) also reported that the hyphae of Cordyceps sinensis as aerial, cottony white to creamish or yellowish, septate, branched and fast growing. Pathania et al. (2015) reported that in Cordyceps militaris the hyphae were thin-walled, branched around $14.8-16.1 \mu \mathrm{m}$ broad.

The conidia of the anamorph were formed either singly or in chains on conidiophores. The conidiophores were observed as single or branched arising from synnemata. The conidia were hyaline, dimorphic having two shapes, viz. spherical and spindle shaped measuring 1.9 to $5.7 \mu \mathrm{m}$ in diameter and 2.5 to $6.3 \mu \mathrm{m}$ in length and 0.53 to $2.41 \mu \mathrm{m}$ in width. Shin et al. (2004) also got matching observations and reported that the imperfect forms of Ophiocordyceps produced spherical or spindle shaped conidia on phialides. Shreshta et al. (2005) reported that in Cordyceps militaris, the shape of the first formed conidia was cylindrical or clavate followed by globose and ellipsoidal.

Perithecia were peripheral to slightly immersed globular to oval in shape, narrow towards the top with an ostioles at the tip. The length of the perithecia was $82.6-396 \mu \mathrm{m}$ and the width was 93.6-171.27 $\mu \mathrm{m}$. Numerous elongated asci containing filiform ascospores which were four-partite and eight in numbers were observed within the perithecia. The size of the ascus was about 115.4-170.11 $\times 5.2-10 \mu \mathrm{m}$ and the ascospore was 105.3-135.7 $\times 2.51-2.73 \mu \mathrm{m}$. Characters of the perithecium in Ophiocordyceps reported by many scientists are in accordance with the above observations. Gwangpo (2000) reported the perithecium of $C$. sinensis as either ovalshaped or egg-shaped, consisting of numerous numbers of thin, long asci containing ascospores. Shin et al. (2004) also observed that the apex of the stroma contains numerous perithecia with several ascospores inside asci. The ascospores were characterized by a thread-like structure in the middle with non disarticulating part spores attached on both sides. Sung et al. (2007) observed perithecia of Ophiocordyceps as superficial to completely immersed, ordinal or oblique in arrangement. Asci were hyaline, cylindrical and ascospores were cylindrical, multiseptate, disarticulating into partspores or non-disarticulating. Observations of Xie et al. (2010) and Zhong et al. (2010) were also similar to the above study, where they found thread like ascospores in ascus. Sung et al. (2010) while working with Cordyceps cardinalis observed that the perithecia were ovoid in shape and semiimmersed on the stroma and the ascospores were unevenly septate but do not disarticulate into part-spores.

The fungus was isolated on PDA medium from the fruiting bodies collected during the survey. This showed that the Potato Dextrose Agar, which is the most widely used medium for fungal isolation can be used for isolation of Ophiocordyceps. Isolation in PDA medium was already reported by many scientists like Sung et al. (2011), Sangdee and Sangdee (2013) and Ko et al. (2017).

Molecular identification of the isolate was done by comparison of ITS sequences at species level. Sequence analysis of the culture showed homology with Ophiocordyceps neovolkiana strain $\mathrm{KC1}$ having 98\% identity. O. neovolkiana strain $\mathrm{KC} 1$ was reported from Tamil Nadu by Chinnusamy and Krishnamoorthi in 2017 (NCBI GenBank: http://ncbi.nlm.nhm.gov/blast). Hence the present study was the first report of $O$. neovolkiana from Kerala.

\section{CONCLUSION}

The fungus was isolated from stroma, stipe and sclerotia of fungal-coconut root grub complex on PDA media followed by periodic sub culturing and maintenance of the isolate in PDA slants under refrigerated condition for further studies. Detailed morphological characterization of the isolate was done. The isolate was identified at species level as Ophiocordyceps neovolkiana. Further studies have to be carried out to unravel the medicinal properties of this fungus and there should be investigations on anti-fungal, antibacterial and biocontrol properties along with detailed studies on mass production of this precious fungus.

\section{ACKNOWLEDGEMENTS}

Authors are grateful to Kerala Agricultural University, Thrissur, Kerala, India for providing facilities to carry out this study in the Department of Plant Pathology at College of Horticulture, Vellanikkara and College of Agriculture, Padannakkad.

\section{REFERENCES}

Arora, R. K. 2015. Cordyceps sinensis (Berk.) Sacc.- an entomophagous medicinal fungus-a review. Int. $J$. Adv. Multidiscip. Res. 2: 0161-0170.

Arora, R. K., Singh, N. and Singh, R. P. 2013. Characterization of an entomophagous medicinal fungus Cordyceps sinensis (Berk.) Sacc. of Uttarakhand, India. The bioscan 8: 195-200.

Chen, J., Zang, W., Lu, T., Zeng, Y., and Kong, L. 2006. Morphological and genetic characterization of a cultivated Cordyceps fungus and its polysaccharaide component possessing antioxidant property in $\mathrm{H} 22$ tumor- bearing mice. Life Sci. 78: 2742-2748.

Chinnusamy, S. and Krishnamoorthy, A. S. 2017. Identification of 3 'deoxyadenosine (Cordycepin) 
from the medicinal mushrooms, Ophiocordyceps spp. Int.j. chem. stud. 5 (3): 788-792.

Dattaraj, H. R., Jagadish, B. R., Sridhar, K. R. and Ghate, S. D. 2018. Are the scrub jungles of Southwest India potential habitats of Cordyceps? Kavaka 51:20-22.

Gwangpo, K. 2000. Characteristics of Cordyceps species. Int . J. Med. Mushrooms 11(5): 537-539.

Holliday, J. and Cleaver, M. 2004. On the Trail of the Yak: Ancient Cordyceps in the modern world. Online posting.

Ko, Y. F., Liau, J. C., Lee, C. S., Chiu, C. Y., Martel, J., Lin, C. S., Tseng, S. F., Ojcius, D. M., Lai, H. C. and Young, J. D. 2017. Isolation, culture and characterization of Hirsutella sinensis mycelium from caterpillar fungus fruiting body. Plos One 12 (1):1-21.

Kobayasi, Y. and Shimizu, D. 1980. Cordyceps species from Japan. 2. Bulletin of the National Science Museum. Series B: Botany: 77-96.

Kumar, S. T. and Aparna, N. S. 2014. Cordyceps species as a bio-control agent against coconut root grub, Leucopholis coneophora Burm. J. Environ. Res. Dev. 8(3A): 614-618.

Li, Y., Wang, X. L., Jiao, L., Jiang, Y., Li, H., Jiang, S. P., Lhosumtseiring, N., Fu, S. Z., Dong, C. H., Zhan, Y. and Yao, Y.J. 2011. A survey of the geographic distribution of Ophiocordyceps sinensis. $J$. Microbiol. 49 (6): 913-919.

Nam, Sung-Hee, Li, Chun-Ru, Hong, In-Pyo, Sung, KyuByoung, Kang, Seok-Woo, Fan, Mei-Zhen, Li, and Zeng-Zhi. 2006. Isolation and Culture of Entomopathogenic Fungus, Cordyceps sphecocephala.Int. J. Ind. Entomol.13 (2): 57-61.

Pathania, P., Joshi, M. and Sagar, A. 2015. Morphological, Physiological and molecular studies on wildly collected Cordyceps militaris from North West Hlmalayas, India. Eur. J. Biotechnol. Biosci. 3 (1): 53-62.

Sangdee, A. and Sangdee, K. 2013. Isolation, identification, culture and production of adenosine and cordycepin from cicada larva infected with entomopathogenic fungi in Thailand. Afr. J.Microbiol. Res. 7 (2): 137146.
Sharma, S. 2004. Trade of Cordyceps sinensis from high altitudes of the Indian Himalaya: Conservation and biotechnological priorities. Curr. Sci. 86:16141619.

Shin, J. C., Shrestha, B., Lee, W. H., Park, Y. J., Kim, S. Y., Jeong, G. R., Kim, H. K., Kim, T. W. and Sung, J. M. 2004. Distribution and favorable conditions for mycelial growth of Cordyceps pruinosa in Korea. Korean J. Mycol. 32 (2): 79-88.

Shrestha, B., Han, S. K., Yoon, K. S. and Sung, J. M. 2005. Morphological Characteristics of Conidiogenesis in Cordyceps militaris. Mycobiol.33 (2): 69-76.

Shrestha, B., Weimin, Z., Yongjie, Z. and Xingzhong, L. 2010. What is the Chinese caterpillar fungus Ophiocordyceps sinensis (Ophiocordycipitaceae). Mycol. Int. J. Fungal Biol. 1 (4): 228-236.

Sridhar, K. R. and Karun, N. C. 2017. Observations on Ophiocordyceps nutans in the Western Ghats. $J$. New Biol. Reports 6 (2): 104-111

Sung, G. H., Hywel-Jones, N. L., Sung, J. M., Luangsa-Ard, J. J., Shrestha, B. and Spatafora, J. W. 2007. Phylogenetic classification of Cordyceps and the clavicipitaceous fungi. Stud. Mycol. 57: 559.

Sung, G. H., Shrestha, B., Han, S. K. and Sung, J. M. 2011. Cultural Characteristics of Ophiocordyceps heteropoda Collected from Korea. Mycobiol.39 (1): 1-6.

Sung, G., Shrestha, B., Han, S. K., Kim, S. Y. and Sung, J. M. 2010. Growth and Cultural Characteristics of Cordyceps cardinalis Collected from Korea. Mycobiol.38(4): 274-281.

Xie, F., Zhu, Z. X., Wei, K. L., Zhang, S. X., Zhang, N. and Tian, W. 2010. Effects of different culture media on the hyphal growth of Cordyceps sinensis strains. Chinese J. Microecol. 22 (6): 534-536.

Zhong, X., Peng, Q. Y., Qi, L. L., Lei, W. and Liu, X. 2010. rDNAtargeted PCR primers and FISH probe in the detection of Ophiocordyceps sinensis hyphae and conidia. J. Microbiol. Methods 83 (2): 188-193. 